\title{
microRNA-146a targets the $L 1$ cell adhesion molecule and suppresses the metastatic potential of gastric cancer
}

\author{
ZHIBO HOU $^{1 *}$, HAITAO YIN $^{1 *}$, CONG CHEN $^{2 *}$, XINZHENG DAI $^{3}$, \\ XIAOLIN $\mathrm{LI}^{4}$, BAORUI LIU ${ }^{5}$ and XUEFENG FANG ${ }^{6}$
}

\author{
${ }^{1}$ Department of Oncology, The Affiliated Zhongda Hospital of Southeast University; ${ }^{2}$ Department of Gynecology of \\ Traditional Chinese Medicine, Jiangsu Provincial Hospital of Traditional Chinese Medicine Affiliated to Nanjing University of \\ Traditional Chinese Medicine; ${ }^{3}$ Department of Liver Surgery, Liver Transplantation Center of Jiangsu Province, \\ The First Affiliated Hospital of Nanjing Medical University; ${ }^{4}$ Department of Geriatrics, The First Affiliated Hospital of \\ Nanjing Medical University, Nanjing, Jiangsu 210029; ${ }^{5}$ The Comprehensive Cancer Center of Drum Tower Hospital, \\ Nanjing University, Nanjing, Jiangsu 210008; ${ }^{6}$ Department of Medical Oncology, Cancer Institute, \\ The Second Affiliated Hospital, Zhejiang University School of Medicine, \\ Hangzhou, Zhejiang 310000, P.R. China
}

Received January 22, 2012; Accepted April 19, 2012

DOI: 10.3892/mmr.2012.946

\begin{abstract}
Recent studies have shown that microRNA-146a (miR-146a) is associated with cancer metastasis. However, the mechanisms underlying this process remain poorly understood. In this study, we aimed to investigate the potential role of miR-146a in gastric cancer metastasis. A wound-healing assay and a Transwell assay were used to investigate the impact of miR-146a on the migratory and invasive abilities of MKN-45 cells in vitro. MKN-45 cells stably expressing miR-146a or the negative control were transplanted into nude mice through the lateral tail vein to explore the effect of miR-146a on tumor metastasis in vivo. A luciferase reporter assay and western blot analysis were used to identify the potential target genes. Our results show that the overexpression of miR-146a inhibits the invasion and metastasis of MKN-45 cells in vitro and in vivo. Furthermore, the L1 cell adhesion molecule (L1CAM) was identified as a novel target of miR-146a in gastric cancer.
\end{abstract}

Correspondence to: Dr Baorui Liu, The Comprehensive Cancer Center of Drum Tower Hospital, Nanjing University, 321 Zhongshan Road, Nanjing 210008, P.R. China

E-mail: baoruiliunj@hotmail.com

Dr Xuefeng Fang, Department of Medical Oncology, Cancer Institute (Key Laboratory of Cancer Prevention and Intervention, China National Ministry of Education), The Second Affiliated Hospital, Zhejiang University School of Medicine, 88 Jiefang Road, Hangzhou, Zhejiang 310000, P.R. China

E-mail: xffang@zju.edu.cn

*Contributed equally

Key words: gastric cancer, microRNA-146a, invasion, metastasis, $\mathrm{L} 1$ cell adhesion molecule
Taken together, our results provide evidence that miR-146a suppresses gastric cancer cell invasion and metastasis in vitro and in vivo, which may be in part due to the downregulation of L1CAM. miR-146a may have the therapeutic potential to suppress gastric cancer metastasis.

\section{Introduction}

According to various cancer statistics over the past decade, gastric cancer remains the fourth most common human epithelial malignancy and is the second leading cause of cancer-related mortality worldwide, with particularly high incidences and mortality rates in eastern Asia $(1,2)$. There are 400,000 new cases of gastric cancer and 300,000 mortalities annually in China (3). Metastasis is the biggest obstacle to the treatment and satisfactory prognosis of gastric cancer. Therefore, the exploration of new therapeutic targets to prevent the metastasis of gastric cancer is urgently required.

An increasing amount of evidence has suggested that microRNAs (miRNAs), which mediate the post-transcriptional regulation of gene expression, control tumorigenesis and cancer metastasis $(4,5)$. The overexpression of oncogenic miRNAs or the underexpression of tumor suppressor miRNAs play critical roles in cancer metastasis.

The dysregulation and dysfunction of miR-146a has been reported to be involved in the development and progression of various types of cancer (4,6-13). We previously found that the expression of miR-146a is downregulated in gastric cancer, and that the downregulation of miR-146a is associated with tumor size, cell differentiation and poor prognosis (14). In addition, miR-146a has been identified as a metastasis-suppressor miRNA in breast cancer and pancreatic cancer (9-11). Recent studies have shown that lower levels of miR-146a are associated with lymph node metastasis and venous invasion in gastric cancer $(15,16)$. However, the mechanisms underlying this process remain poorly understood. 
In the current study, the role of miR-146a in gastric cancer metastasis was investigated. The results from a wound-healing assay and a Transwell assay in vitro and a metastasis formation assay in vivo demonstrated that miR-146a suppresses gastric cancer cell invasion and metastasis. Furthermore, a luciferase reporter assay and western blot analysis were used to confirm that miR-146a functions as a metastatic suppressor in gastric cancer by targeting the L1 cell adhesion molecule (L1CAM) directly.

\section{Materials and methods}

Cell lines and culture conditions. The human gastric cancer cell line, MKN-45, was obtained from the Shanghai Institute of Cell Biology (Shanghai, China). The cells were propagated in RPMI-1640 medium (Invitrogen, Carlsbad, CA, USA), supplemented with $10 \%$ fetal bovine serum (FBS). The cells were cultured at $37^{\circ} \mathrm{C}$ in a water-saturated atmosphere with $5 \% \mathrm{CO}_{2}$.

Transient overexpression of miR-146a. miR-146a mimics and negative control mimics were obtained from Shanghai GenePharma Co. Ltd. (Shanghai, China) (14). miR-146a mimics and negative control mimics were transfected into MKN-45 cells with siPORT ${ }^{\mathrm{TM}} \mathrm{NeoFX}^{\mathrm{TM}}$ Transfection Agent (Ambion, Austin, TX, USA) according the manufacturer's instructions, at a final concentration of $50 \mathrm{nM}$.

Scratch wound healing assay. A scratch wound healing assay was performed as previously described (17). Briefly, transfected cells in 6-well plates were cultured until cells reached confluency, and were then starved overnight. Cell layers were wounded using a $200 \mu \mathrm{l}$ pipette tip and cultured for another $48 \mathrm{~h}$. Photographs were taken at the 0-, 24- and 48-h timepoints.

Cell migration and invasion assay. A Transwell cell migration and Matrigel invasion assay were carried out to investigate the impact of miR-146a on the migratory and invasive ability of MKN-45 cells. For migration detection, transfected cells were placed in a Transwell chamber at $2 \times 10^{4}$ cells/well. The lower Transwell chamber contained 10\% FBS for use as a chemoattractant. For the invasion assay, the bottom of the culture inserts (8-mm pores) were coated with $30 \mu \mathrm{l}$ of the mixture containing serum-free RPMI-1640 and Matrigel (1:8; BD Biosciences, Bedford, MA, USA). The Matrigel was allowed to solidify at $37^{\circ} \mathrm{C}$ overnight. After solidification, cells $\left(2 \times 10^{4}\right.$ cells/well) were re-seeded onto the upper chamber. After $24 \mathrm{~h}$, the cells that had migrated or invaded through the membrane were fixed with $95 \%$ alcohol and stained with crystal violet. The number of migrated cells or invaded cells was quantified by counting 5 independent symmetrical visual fields under the microscope.

Construction of miR-146a expression vectors and cell transfection. The pcDNA6.2-GW/EmGFP-miR plasmid vector (Invitrogen) was used to construct a miR-146a overexpressing plasmid. A DNA fragment with mature miR-146a or a negative control mismatched sequence was chemically synthesized and inserted into this vector. The nucleotide sequences of the inserts were verified to be in the correct sequence and orientation for expression in the new construct by DNA sequencing. The stable transfection of the appropriate miRNA plasmids was carried out using Lipofectamine 2000 (Invitrogen) according to the manufacturer's instructions. Successfully transfected cell clones were obtained in a selection medium containing $3.5 \mu \mathrm{g} /$ $\mathrm{ml}$ blasticidin to generate 2 stable monoclonal cell lines (the miR-146a stable cell line, MKN-45-miR-146a, and the control stable cell line, MKN-45-miR-negative control). Real-time PCR was performed as previously described to detect the expression levels of miR-146a after stable transfection (14).

In vivo metastasis assays. For in vivo metastasis assays, MKN-45-miR-146a cells or MKN-45-miR-negative control (MKN-45-miR-NC) cells were transplanted into nude mice (5-week-old BALB/c-nu/nu, 10 per group, $1 \times 10^{6}$ cells for each mouse) through the lateral tail vein. Mice were sacrificed after 10 weeks. All animal experiments were performed in full compliance with previously established guidelines approved by the Animal Care Committee at Drum Tower Hospital. The lungs were dissected and subjected to hematoxylin \& eosin (H\&E) staining. The number of metastases in the lungs was examined histologically.

Luciferase reporter assay. For the luciferase reporter experiment, a 3'-UTR segment containing the miR-146a-binding site of L1CAM was chemically synthesized and inserted into the pGL3-control vector (Promega, Madison, WI, USA) at the $X b a I$ site immediately downstream from the luciferase stop codon. DNA segments with scrambled target sites (L1CAM) designed to interfere with seed sequence recognition were also cloned to serve as a control for specificity. In total, $800 \mathrm{ng}$ of the firefly luciferase reporter vector and $80 \mathrm{ng}$ of the control vector containing Renilla luciferase, pRL-TK (Promega), were transfected in combination with $50 \mathrm{pM}$ miR-146a or negative control using Lipofectamine 2000 in 24-well plates according to the manufacturer's instructions. Luciferase activities were measured $24 \mathrm{~h}$ after transfection using the Dual Luciferase Reporter assay system (Promega) on a Centro LB 960 (Berthold, Bad Wildbad, Germany). Three independent experiments were performed in triplicate.

Western blot analysis. For protein extraction, confluent cells were washed with cold PBS twice, denatured in lysis buffer (20 mM Tris- $\mathrm{HCl}, 200 \mathrm{mM} \mathrm{NaCl}, 0.2 \%$ Nonidet P-40, 0.5\% Triton X-100 and protease inhibitors) and boiled at $100^{\circ} \mathrm{C}$ for $10 \mathrm{~min}$. Equal amounts of protein extracts were separated on $10 \%$ polyacrylamide gels by using standard sodium dodecyl sulfate-polyacrylamide gel electrophoresis (SDS-PAGE), then transferred onto PVDF membranes (Bio-Rad, Hercules, CA, USA). Following blocking in Tris-buffered saline (TBS) with $0.1 \%$ Triton X-100 and 5\% milk, the membranes were incubated with mouse anti-human L1CAM monoclonal antibody (Santa Cruz, CA, USA) and anti- $\beta$-actin (Sigma-Aldrich, St. Louis, MO, USA) at $4^{\circ} \mathrm{C}$ overnight. Following washing, the membranes were incubated with secondary goat anti-mouse antibody conjugated to horseradish peroxidase (SigmaAldrich) at room temperature for $1 \mathrm{~h}$. Signal detection was carried out by an enhanced chemiluminescence (ECL) system (Amersham Pharmacia Biotech). 

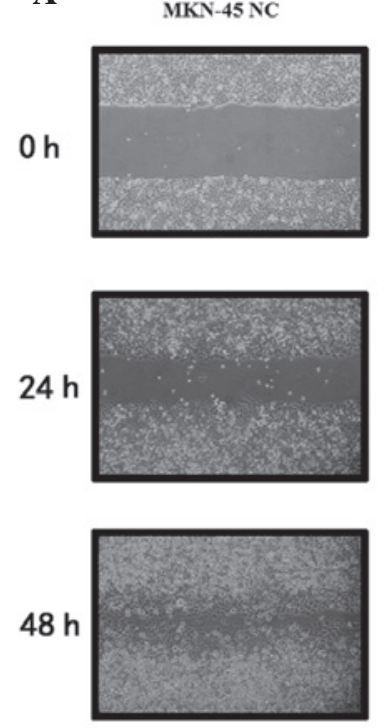

MKN-45 miR-146a
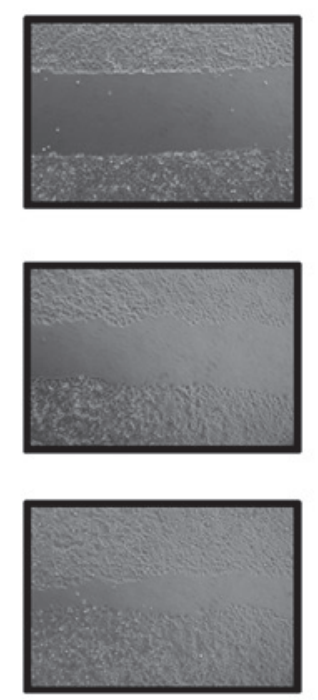

B

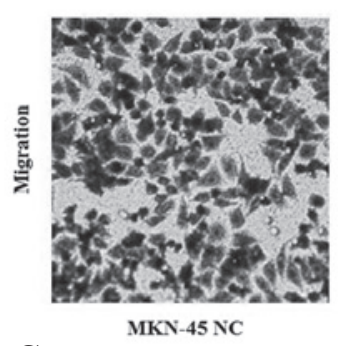

C

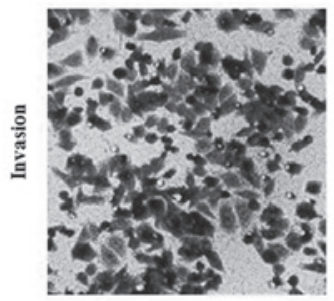

MKN-45 NC

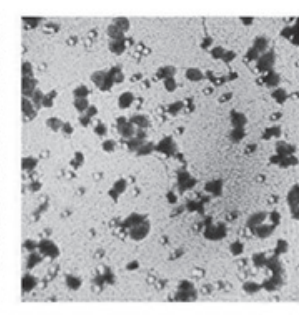

MKN-45 miR-146a

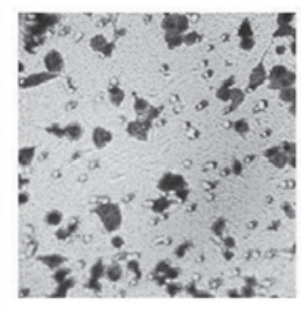

MKN-45 miR-146a

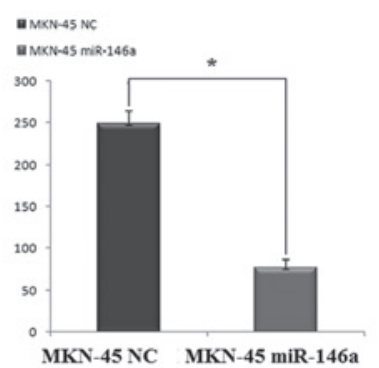

MKN-45 NC MKN-45 miR-146a

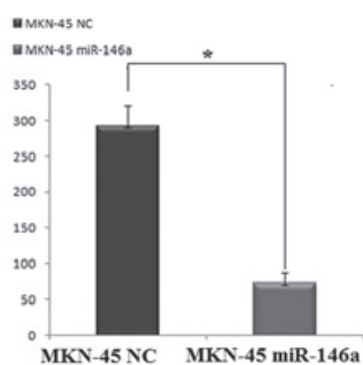

Figure 1. Overexpression of miR-146a on cell migration and invasion in vitro. (A) Wound healing assays were used to evaluate the motility and migration of MKN-45 cells transfected with miR-146a mimics or the negative controls. Images were captured at 0, 24 and $48 \mathrm{~h}$ post-wounding. (B) Representative photomicrographs of Transwell results for MKN-45 cells were taken under x100 original magnification. The number of MKN-45 cells transfected with miR-146a mimics passing through the membrane without Matrigel was significantly lower than that of MKN-45 cells transfected with negative controls. (C) The number of MKN-45 cells transfected with miR-146a mimics passing through the membrane with Matrigel was significantly lower than that of MKN-45 cells transfected with negative controls. "Denotes statistically significant differences between the groups $(\mathrm{P}<0.01)$. NC, negative control.

Statistical analysis. Each experiment was repeated at least 3 times. All results are expressed as the means \pm standard deviation (SD). The difference between the means was analyzed using the Student's t-test or the $\chi^{2}$ test. All statistical analyses were performed using SPSS 16.0 software (Chicago, IL, USA). Differences were considered significant when $\mathrm{p}<0.05$.

\section{Results}

miR-146a suppresses gastric cancer migration and invasion in vitro. A wound-healing assay was performed to examine the effect of overexpression of miR-146a on cell migration. It was found that MKN-45 cells transfected with miR-146a mimics closed the scratch wound more slowly than cells transfected with negative controls (Fig. 1A). We also estimated the effects of miR-146a on the migration and invasion of MKN-45 cells using a Transwell cell migration and Matrigel invasion assay. The data demonstrated that the overexpression of miR-146a markedly inhibited the migration and invasion of MKN-45 cells. The number of MKN-45 cells transfected with miR146a mimics $(292 \pm 29, \mathrm{P}<0.01)$ that had migrated through the membrane without Matrigel was significantly lower than that of MKN-45 cells transfected with the negative controls $(72 \pm 15)$ (Fig. 1B). A similar result was found with the invaded cells; the number of MKN-45 cells transfected with miR-146a mimics $(249 \pm 16, \mathrm{P}<0.01)$ passing through the Matrigel was significantly lower than that of MKN-45 cells transfected with the negative controls $(77 \pm 10)$ (Fig. 1C).

miR-146a suppresses gastric cancer cell metastasis in vivo. To further explore the effects of miR-146a on tumor metastasis in vivo, plasmids stably expressing miR-146a or the negative control were constructed (Fig. 2A). miR-146a or negative control plasmids were then stably transfected into human gastric cancer MKN-45 cells. As shown in Fig. 2B, miR-146a levels were higher in the cells stably expressing miR-146a than in the cells transfected with the negative control. MKN-45-miR-146a cells or MKN-45-miR-NC cells were transplanted into nude mice through the lateral tail vein. Histological analysis of the lungs of mice confirmed that miR-146a suppressed lung metastasis formation. Lung metastasis of gastric cancer was apparent in mice injected with MKN-45-miR-NC cells (Fig. 3). By contrast, few metastatic tumors were detected in the mice injected with MKN-45-miR-146a (Fig. 3). Our results indicate that miR-146a is a negative regulator for gastric cancer metastasis.

miR-146a post-transcriptionally reduces L1CAM expression by directly targeting its 3'UTR. For miRNA target prediction, we used microrna.org online software (http://www.microrna. org/microrna/getMirnaForm.do). Among a total of 6,798 genes that were potentially targeted by miR-146a, it was found that L1CAM may contribute to the metastasis of gastric cancer (Fig. 4A). The overexpression of L1CAM has been correlated with tumor progression and metastasis of a number of types of cancer, including gastric cancer $(18,19)$. To confirm that L1CAM is a direct target of miR-146a, we constructed the luciferase reporter, pGL3-L1CAM-3'UTR. A scrambled target site (pGL3-L1CAM-MUT) was also constructed as the control for sequence specificity. All the reporters were transfected in MKN-45 cells. In MKN-45 cells, a significant decrease in relative luciferase activity was noted when pGL3-L1CAM-3'UTR was co-transfected with the mature miR-146a mimic but not with the negative control (Fig. 4B). On the contrary, there was no significant difference of the relative luciferase activity of the pGL3-L1CAM-MUT reporter between MKN-45 cells transfected with miR-146a mimics and MKN-45 cells trans- 
A

miR-146a 5'-TGCTGtgagaactgaattccatgggttGTTTTGGCCACTGACTGACaacccatgattcagttetca-3' 3'-CactcttgacttaaggtacccaaCAAAACCGGTGACTGACTG ttgggtactaagtcaagagt-5'

$\mathrm{NC}$ 5'-TGCTGaaatgtactgegcgtggagacGTTITGGCCACTGACTGACgtctcacgcagtacattt-3' 3'-CtttacaggacgcgcactetgCAAAACCGGTGACTGACTGcagaggtgcgtcatgtaaa-5'

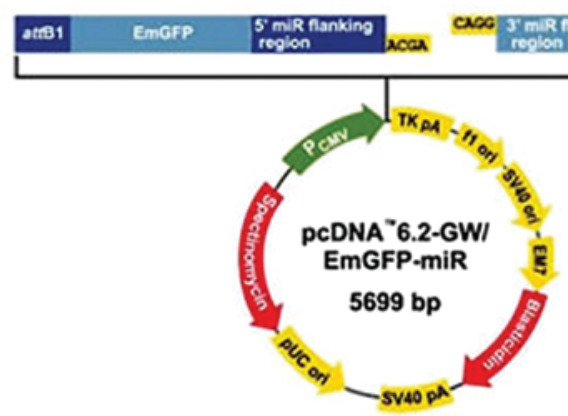

70

80

90

100

110

120

I G AGAACI GAAI CAI G G GI IG ICA G I CA GI G GC CAAAACAA CC CA I G G AAI I CA G I ICICACA GCA.

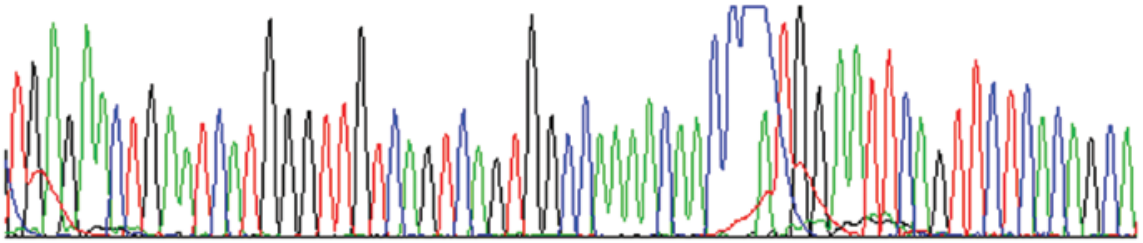

B

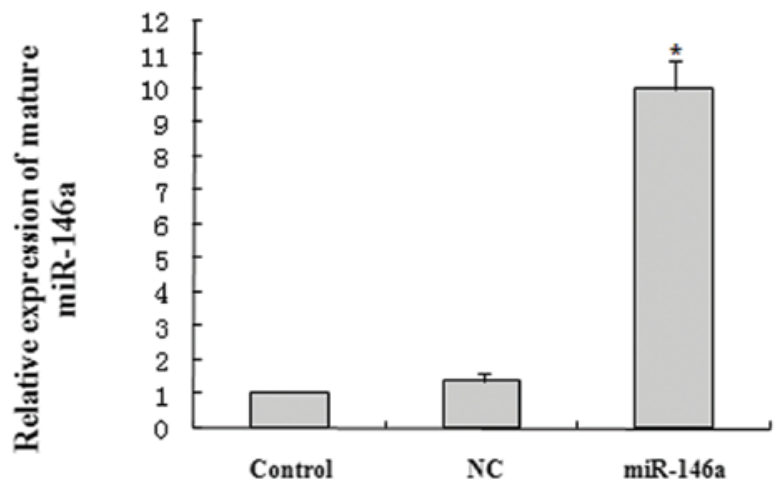

Figure 2. miR-146a construction. (A) The sequence of the DNA fragment with mature miR-146a or the negative control. A DNA fragment with mature miR-146a or the negative control mismatched sequence was chemically synthesized and inserted into a pcDNA6.2-GW/EmGFP-miR plasmid. miR-146a was confirmed by automated sequencing. (B) Following stable transfection of miR-146a, RT-PCR confirmed that miR-146a levels were higher in cells stably expressing miR-146a than in cells transfected with the negative control. ${ }^{*}$ Denotes statistically significant differences between the groups $(\mathrm{P}<0.01)$. NC, negative control.

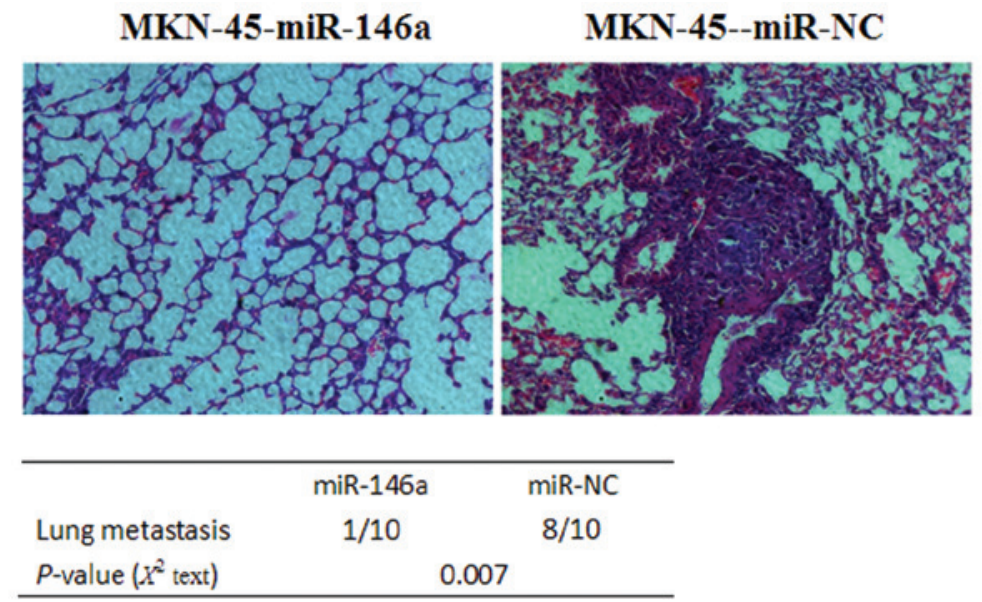

Figure 3. miR-146 suppresses gastric cancer metastasis in vivo. Representative H\&E stained sections of the lung tissues isolated from mice implanted with MKN45-miR-146a cells or MKN-45-miR-NC cells through the lateral tail vein. The data shown are the number of lung metastases from each group. H\&E, hematoxylin \& eosin; NC, negative control. 
A

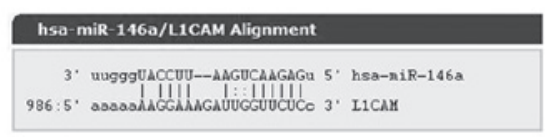

B

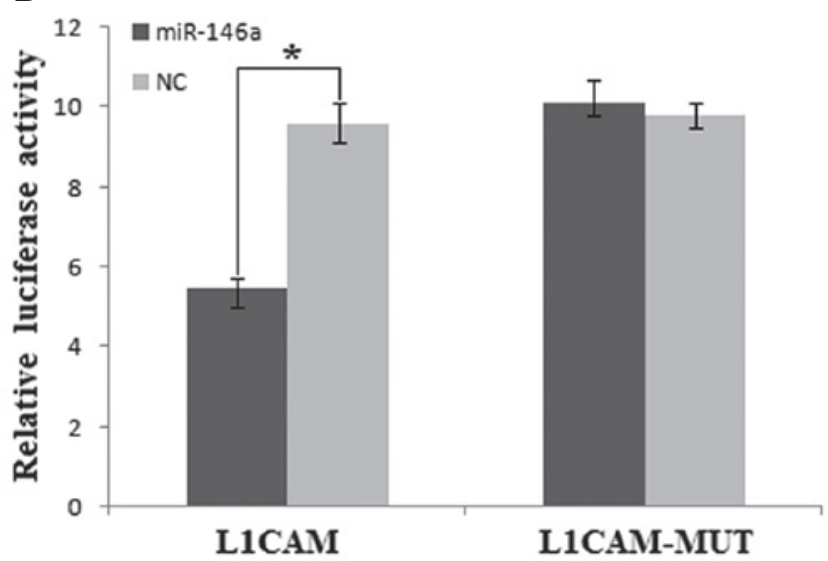

C

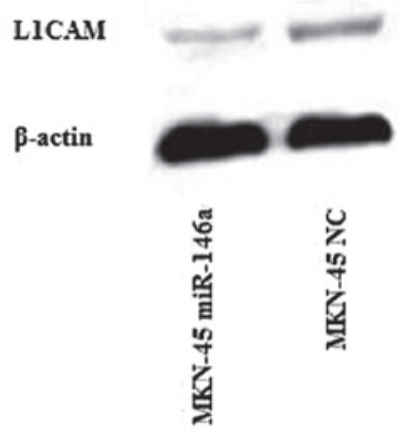

Figure 4. L1CAM is the potential target of miR-146a in gastric cancer MKN-45 cells. (A) The target site of miR-146a in the 3'UTR of L1CAM by microrna. org online software. (B) Analysis of relative luciferase activity of L1CAM and L1CAM-MUT in MKN-45 cells transfected with miR-146a mimics or negative controls (NC). "Denotes statistically significant differences between the groups $(\mathrm{P}<0.01)$. (C) Protein expression analysis for L1CAM in MKN-45 cells transfected with miR-146a mimics or negative controls by western blot analysis. L1CAM, L1 cell adhesion molecule; NC, negative control.

fected with negative controls (Fig. 4B). These results suggest that miR-146a downregulates L1CAM expression by directly targeting its 3'UTR. In support of these results, we next examined the L1CAM protein level in MKN-45 cells transfected with miR-146a mimics or negative controls by western blot analysis. A clear reduction in the level of the endogenous L1CAM protein in miR-146a-transfected MKN-45 cells was observed compared to the negative control-transfected cells normalized to an endogenous reference $\beta$-actin protein (Fig. 4C). These results demonstrate that miR-146a targets L1CAM in gastric cancer.

\section{Discussion}

Previous reports have shown that miR-146a may function as a tumor suppressor or oncogene, depending on the tumor type. The downregulation of miR-146a is a frequent event in various types of tumors, such as natural killer T-cell lymphoma, glioma, prostate and gastric cancer $(7,10,11,14,15)$. On the contrary, miR146a is upregulated in cervical cancer and anaplastic thyroid carcinoma and functions as an oncogene $(6,13)$. The role of miR-146a in gastric cancer is inconsistent. We, and others, have shown previously that miR-146a is downregulated in gastric cancer and may function as a tumor suppressor (14-16), while there is a contradictory result that miR-146a is upregulated in gastric cancer (20). In this study, we further show that the overexpression of miR-146a suppressed gastric cancer MKN-45 cell invasion and metastasis in vitro and in vivo. L1CAM was identified as a direct target of miR-146a. The data from the current study suggest that miR-146a acts as a potential metastasis suppressor in gastric cancer.

Our findings are consistent with those from recent reports showing that miR-146a suppresses the metastasis of many types of cancer including pancreatic, breast and prostate cancer $(4,7,9,12)$. More recently, Kogo et al reported that the ectopic expression of miR-146a inhibits the migration and invasion of gastric cancer in vitro (15). These results collectively suggest that miR-146a functions as a metastasis-suppressor miRNA in gastric cancer.

miR-146a is commonly lost in metastatic prostate cancer and the downregulation of miR-146a may be the late event in the progression of prostate cancer $(5,7)$. Lin et al found that miR-146a was expressed markedly in non-cancerous prostatic epithelium and gradually disappeared with cancer progression (7). If the loss of miR-146a expression is also a late event in the progression of gastric cancer, based on our results that miR-146a is a metastasis-suppressor miRNA in gastric cancer, the loss of miR-146a expression may lead to increased invasiveness and metastatic ability in gastric cancer. Therefore, the expression of miR-146a may correlate with the metastatic ability and clinical stage of gastric cancer. The tissue samples used in the different studies perhaps account for the contradictory results; certain studies have shown that miR-146a expression is downregulated, while others have shown that it is upregulated. Indeed, Kogo et al also reported that the miR-146a level was associated with the clinical stage (TNM) (15). We speculate that miR-146a may exert its bidirectional function in gastric cancer and that miR-146a expression may be lost gradually in the late stage of gastric cancer, thus promoting cancer metastasis. A larger number of tissue samples are still required to confirm this in future studies. Although the expression of miR-146a is contradictory in gastric cancer, there is no disagreement as to the function of miR-146a as a metastasis-suppressor. miR-146a may be a new target for suppressing tumor metastasis.

Previous studies have found that miR-146a inhibits tumor metastasis by directly targeting the epidermal growth factor receptor (EGFR), interleukin 1 receptor-associated kinase 1 (IRAK1), TNF receptor-associated factor 6 (TRAF6), Notch1 and Rho-associated, coiled-coil-containing protein kinase 1 (ROCK1) in pancreatic, breast, glioma and prostate cancer $(4,9,10,12)$. Kogo et al reported that miR-146a inhibits migration and invasion and downregulates EGFR and IRAK1 expression in vitro (15). In the current study, we found a novel direct target of miR-146a,L1CAM. We used a luciferase reporter assay and western blot analysis to confirm that L1CAM is a target of miR-146a in gastric cancer cells. L1CAM is initially identified in the nervous system and is a cell adhesion molecule which belongs to the immunoglobulin superfamily of cell adhesion molecules (IgCAM) (21). It has been observed exclusively 
in the invasion front of colorectal cancer (22). L1CAM expression in gastric cancer is a relatively strong prognostic factor for patients with pathologically confirmed T3-stage cancer (19).

In conclusion, the results from the present study show that miR-146a suppresses gastric cancer cell invasion and metastasis, which was in part due to the downregulation of L1CAM, the molecular target that is commonly associated with gastric cancer metastasis and prognosis. Therefore, miR-146a may have the therapeutic potential to suppress gastric cancer invasion and metastasis.

\section{Acknowledgements}

This study was supported by the National Natural Science Foundation of China (No. 81101580; 81071815), Jiangsu Province Key Medical Center Foundation and Scientific and Technological Innovation Plan Fund of Postgraduate from Jiangsu Province (No. 5X22013084).

\section{References}

1. Parkin DM, Bray F, Ferlay J and Pisani P: Global cancer statistics, 2002. CA Cancer J Clin 55: 74-108, 2005

2. Leung WK, Wu MS, Kakugawa Y, et al: Screening for gastric cancer in Asia: current evidence and practice. Lancet Oncol 9: 279-287, 2008.

3. Yang L: Incidence and mortality of gastric cancer in China. World J Gastroenterol 12: 17-20, 2006.

4. Bhaumik D, Scott GK, Schokrpur S, Patil CK, Campisi J and Benz CC: Expression of microRNA-146 suppresses NF- $\mathrm{B}$ activity with reduction of metastatic potential in breast cancer cells. Oncogene 27: 5643-5647, 2008.

5. Nicoloso MS, Spizzo R, Shimizu M, Rossi S and Calin GA: MicroRNAs - the micro steering wheel of tumour metastases. Nat Rev Cancer 9: 293-302, 2009.

6. Wang X, Tang S, Le SY, et al: Aberrant expression of oncogenic and tumor-suppressive microRNAs in cervical cancer is required for cancer cell growth. PLoS One 3: e2557, 2008.

7. Lin SL, Chiang A, Chang D and Ying SY: Loss of mir-146a function in hormone-refractory prostate cancer. RNA 14: 417-424, 2008

8. Jazdzewski K, Murray EL, Franssila K, Jarzab B, Schoenberg DR and de la Chapelle A: Common SNP in pre-miR-146a decreases mature miR expression and predisposes to papillary thyroid carcinoma. Proc Natl Acad Sci USA 105: 7269-7274, 2008.
9. Hurst DR, Edmonds MD, Scott GK, Benz CC, Vaidya KS and Welch DR: Breast cancer metastasis suppressor 1 up-regulates miR-146, which suppresses breast cancer metastasis. Cancer Res 69: 1279-1283, 2009.

10. Mei J, Bachoo R and Zhang CL: MicroRNA-146a inhibits glioma development by targeting Notch1. Mol Cell Biol 31: 3584-3592, 2011.

11. Paik JH, Jang JY, Jeon YK, et al: MicroRNA-146a downregulates NFKB activity via targeting TRAF6 and functions as a tumor suppressor having strong prognostic implications in NK/T cell lymphoma. Clin Cancer Res 17: 4761-4771, 2011.

12. Li Y, Vandenboom TG, 2nd, Wang Z, et al: miR-146a suppresses invasion of pancreatic cancer cells. Cancer Res 70: 1486-1495, 2010.

13. Pacifico F, Crescenzi E, Mellone S, et al: Nuclear factor- $\kappa \mathrm{B}$ contributes to anaplastic thyroid carcinomas through up-regulation of miR-146a. J Clin Endocrinol Metab 95: 1421-1430, 2010.

14. Hou Z, Xie L, Yu L, Qian X and Liu B: MicroRNA-146a is downregulated in gastric cancer and regulates cell proliferation and apoptosis. Med Oncol 29: 886-892, 2012.

15. Kogo R, Mimori K, Tanaka F, Komune S and Mori M: Clinical significance of miR-146a in gastric cancer cases. Clin Cancer Res 17: 4277-4284, 2011.

16. Tchernitsa O, Kasajima A, Schafer R, et al: Systematic evaluation of the miRNA-ome and its downstream effects on mRNA expression identifies gastric cancer progression. J Pathol 222: 310-319, 2010.

17. Liang CC, Park AY and Guan JL: In vitro scratch assay: a convenient and inexpensive method for analysis of cell migration in vitro. Nat Protoc 2: 329-333, 2007.

18. Raveh S, Gavert N and Ben-Ze'ev A: L1 cell adhesion molecule (L1CAM) in invasive tumors. Cancer Lett 282: 137-145, 2009.

19. Kodera Y, Nakanishi H, Ito S, et al: Expression of L1 cell adhesion molecule is a significant prognostic factor in pT3-stage gastric cancer. Anticancer Res 29: 4033-4039, 2009.

20. Xiao B, Zhu ED, Li N, et al: Increased miR-146a in gastric cancer directly targets SMAD4 and is involved in modulating cell proliferation and apoptosis. Oncol Rep 27: 559-566, 2012.

21. Rathjen FG and Schachner M: Immunocytological and biochemical characterization of a new neuronal cell surface component (L1 antigen) which is involved in cell adhesion. EMBO J 3: 1-10, 1984.

22. Gavert N, Conacci-Sorrell M, Gast D, et al: L1, a novel target of beta-catenin signaling, transforms cells and is expressed at the invasive front of colon cancers. J Cell Biol 168: 633-642, 2005. 